\title{
Categories as paradigms for comparative cognition
}

\author{
René Zayan ${ }^{\mathrm{a}, *}$, Jacques Vauclair ${ }^{\mathrm{b}}$ \\ a Faculté de Psychologie, Université de Louvain, Place Désiré Mercier, 10, B-1348 Louvain-la-Neuve, Belgium \\ ${ }^{\mathrm{b}}$ Centre de Recherche en Neurosciences Cognitives, CNRS, 31 Chemin Joseph-Aiguier, F-13402 Marseille Cedex 20, France
}

\begin{abstract}
Forming categories is a basic cognitive operation allowing animals to attain concepts, i.e. to represent various classes of objects, natural or artificial, physical or social. Categories can also be formed about the relations holding among these objects, notably similarity and identity. Some of the cognitive processes involved in categorisation will be enumerated. Also, special reference will be made to a much neglected area of research, that of social representations. Here, animals conceive the natural class of their conspecifics as well as the relationships established between them in groups. Two types of social categories were mentioned: (1) intraspecies recognition including recognition of individual conspecifics; and (2) representation of dominance hierarchies and of their transitivity in linear orders. (C) 1998 Elsevier Science B.V.
\end{abstract}

Keywords: Categorisation; Natural concept; Prototype; Social recognition; Individual recognition; Dominance hierarchy; Transitivity

\section{Cognitive degrees of category formation}

The ability to discriminate and assign objects (e.g. visual shapes) to categories is involved in many crucial human cognitive activities such as perception and memorisation (Harnad, 1987). By simplifying the complex and changing environment to which subjects are faced with, this ability makes it possible to considerably reduce the amount of cognitive demand. In addition, the

\footnotetext{
* Corresponding author. Present address: 1-Place Mercier, B-3848 Louvain-la-Neuve, Belgium. Tel.: + 3210 472734; fax: + 3210 473774; e-mail: lempereur@expe.ucl.ac.be
}

classification of a novel item into a category allows subjects to adapt their behaviours by applying to this item their knowledge about the category (generalisation). In other words, using categories implies that subjects have detected some invariant properties of unique objects that are being represented, i.e. conceive some class of supra-objects.

The following sections will propose a summary concerning the available evidence of categorisation behaviours in comparative cognition. This review will concentrate on the different levels of categorisation attained by animals in their abilities to lump together members which share physi- 
cal or conceptual relations. Moreover, it will envisage possible perceptual and cognitive processes that govern these behaviours.

According to Herrnstein (1990), the control of the stimuli that surround an organism is determined by several factors, among which are the physical variations of the stimulus, the sensorimotor equipment of the organism and the functional consequences for the organism in question when a given behaviour is produced. From the interaction of these factors, Herrnstein (1990) has distinguished five levels of stimulus control, or categorisation (ranging from the lowest to the highest level of abstraction): simple discriminations, 'categorisation by rote', open-ended categories, functional categorisation, and categorisation of 'abstract relations'.

The following sections will propose a classificatory scheme of categorical behaviours and representations in animal species. This scheme is broadly based on Herrnstein's levels, but it will also draw upon recent findings related to categories formed for both natural and artificial objects and for abstract or relational concepts (e.g. Roitblat and von Fersen, 1992; Thompson, 1995).

\subsection{Levels of category formation}

\subsubsection{Categorisation by rote}

This level requires discrimination and memorisation of any arbitrary list of stimuli, for which classification criteria are dependent upon contingency rules. The best example of such behaviours is provided by a study on pigeons (Vaughan and Green, 1984). In separate experiments, the birds were tested with two kinds of visual stimuli (arbitrary squiggles and photographs of natural scenes). The pigeons successfully sorted up to 160 exemplars of squiggles and 320 photographs into arbitrary categories. Moreover, some of the pigeons tested one year later performed above chance in categorising the 40 last exemplars of each stimulus type. These capacities are reminiscent of that exhibited by food caching birds which can remember hundreds of food locations (see Kamil and Balda, 1985, for an example concerning Clark's nutcrackers).
Thus, if there is solid experimental evidence that animals can accurately memorise large amounts of information (objects' features, list of spatial locations, etc.), it is notwithstanding obvious that attentional demands as well as memory storage abilities must be enormous to keep track of such information. It is likely that economical principles must be at work to ease these loads. For example, in the realm of spatial memory, serial position effects (primacy and/or recency) influence performance accuracy in retrieving caches (see Crystal and Shettleworth, 1994, for an example with black-capped chickadees).

\subsubsection{Open-ended categories}

Here too, economical rules seem to intervene in object-sorting behaviours. Such rules are based on some principle of 'perceptual similarity' (Herrnstein, 1990, p. 136). Thus, contrary to rote categorisation, open-ended categories rest on a perceptual similarity between individuals that belong to a given class. For example, Guinea baboons vary from one individual to another, but they have features in common which make them recognisable from other baboons belonging to a different subspecies (e.g. hamadryas baboons). This similarity principle acquired with a given set of exemplars should generalise to novel elements of the same kind.

Exploration of this level of categorisation has been inaugurated by the studies carried out with pigeons by Herrnstein and his co-workers (Herrnstein and Loveland, 1964; Herrnstein et al., 1976). Using projected photographs, they demonstrated that, after appropriate training with an operant conditioning procedure, pigeons could accurately classify previously unseen pictures of categories such as humans (Herrnstein and Loveland, 1964), pigeons (Herrnstein, 1990), trees (Herrnstein et al., 1976) or other objects (Delius, 1992; see Watanabe et al., 1993 for a complete overview of category discrimination in birds). The study of categorical abilities has been extended to nonhuman primates (e.g. Schrier et al., 1984; Yoshikubo, 1985; D'Amato and van Sant, 1988; Roberts and Mazmanian, 1988).

At this stage, it is noteworthy that category representations can operate on two different kinds 
of materials, so as to produce on the one hand, natural categories such as trees (e.g. Herrnstein et al., 1976) or monkeys (Schrier et al., 1984) and on the other hand, artificial categories such as the categorical discrimination between the letter ' $A$ ' and the number '2', (for pigeons: Morgan et al., 1976; for monkeys: Schrier et al., 1984; Vauclair and Fagot, 1996).

\subsubsection{Conceptual categorisation}

There are two criteria which are retained by most authors (e.g. Herrnstein, 1990) to define conceptual categorisation. The first criterion is met when a rapid generalisation over class members of classificatory items is observed. The second criterion, related to conceptual processing, implies categorisation abilities that go beyond similarity between exemplars of a class. In this respect, sorting objects on the basis of some functional similarity, e.g. 'food' versus 'tool' (SavageRumbaugh et al., 1980; Gardner and Gardner, 1984) would qualify as conceptual behaviour. It is generally assumed that reference to 'concepts' is made only to emphasise 'the fact that the categories are usually defined in terms of a human language concept' (Watanabe et al., 1993, p. 353).

As noted by Herrnstein (1990) and Thompson (1995), the operational distinction between openended categories and concept is difficult to make, especially since the role of conceptual mediation is difficult to assess, given the still imprecise nature of the mechanisms that control categorical behaviours (see the section on cognitive processes).

\subsubsection{Categorisation of abstract relations}

This categorisation level was originally defined as dealing 'not with the exemplars themselves, but with relations between and among concepts' (Herrnstein, 1990, p. 138), as defined above.

There is a large amount of literature devoted to the mastery of general, abstract relations by animals of different phyla (see examples in Roitblat and von Fersen, 1992; Thompson, 1995). The most widely used methods to demonstrate these abilities are matching-to-sample (MTS) and oddity problems. In a typical experiment of this kind, the animal is trained with a limited number of stimuli (e.g. blue and red objects) and, once per- formance has reached a consistently high level of correct responses, the subject is tested with novel stimuli. Pigeons and monkeys were able to respond to visual stimuli on the basis of a relation of identity, as in colour ('the same colour as', Zentall and Hogan, 1976) or form (D'Amato et al., 1985). A general matching concept has also been found in marine mammals (for an example with auditory and visual stimuli in the dolphin, see Herman et al., 1989).

Acquisition of an identity concept in matchingto-sample can be inferred from successful transfer to novel sample stimuli following acquisition with only two stimuli serving alternatively as the sample and nonmatching choice during training (Oden et al., 1988). If novel stimuli only are used on each trial during training, then the transfer of matching may in fact be mediated on the basis of either absolute or relative familiarity and novelty, rather than on the basis of reflexive identity. That is, a subject may simply recognise that a matching comparison stimulus is familiar rather than explicitly judge it to be identical to the sample (Oden et al., 1990; Thompson and Oden, 1996). Even at the categorical level (e.g. 'match fruit with fruit'), each generalised MTS trial entails just a single identity equivalence judgement. Situations in which MTS is used to test a subject's ability to judge whether 'relations between relations' (Premack, 1983) are equivalent are more complex (Thompson, 1995).

For example, one might test whether a pair of balls is perceived or judged to hold the same relation (identity) as a pair of shoes and whether a paired lock and cup reflects the same relation (non-identity) as a paired pencil and canister (e.g. Oden et al., 1990; Tyrrell et al., 1991). Asked to judge the relationship between identity (paired shoes) and non-identity (lock and cup), one might correctly label them as being different. From a computational stance, successful completion of abstract relational MTS tasks entails, unlike physical or categorical judgements, not one but three initial within-pair identity/non-identity judgements. Subsequently, the coded outcomes of the three initial comparisons must be compared if the correct matching pair is to be identified. 
Such relational requirements have been tested in a conceptual matching-to-sample task (a modified version of an analogy problem) proposed to chimpanzees with previous training with conditional and numerical tokens (Thompson et al., 1997). Four of the five subjects were successful at conceptually matching relations between relations, even though they were provided with no explicit training via differential feedback on each trial. Apparently, such an ability still awaits demonstration in animals species other than chimpanzees (but see Neiworth and Wright, 1994; Cook et al., 1995, for some evidence of the use of a generalised same-different concept in pigeons and monkeys, respectively).

\subsection{The cognitive processes involved and their possible explanations}

A number of perceptual-cognitive processes should in principle correspond to the different levels of categorisation just described. Some of these processes have been identified. The following list is by no means definitive nor exhaustive (see also Watanabe et al., 1993 and Thompson, 1995).

\subsubsection{Absolute discriminations}

It is likely that animals facing categorisation tasks rely on simple perceptual dimensions of the stimuli such as colour, luminance, or other psychophysical attributes. Accordingly, it is important, in all studies aimed at documenting categorical behaviour in animals, to inquire whether discrimination and transfer are mediated by simple low level perceptual processing of the stimuli before advocating the mediation of some higher (e.g. conceptual) processing. There is evidence that animals might respond on the basis of such absolute, sometimes artifactual, cues. Thus, Lea and Ryan (1990) have reported that pigeons might have used the luminosity of the stimuli to solve certain categorisation problems. On other occasions, pigeons might neglect cues that were thought to be very salient in the experimenters' eyes (e.g. in a letters' categorisation task by pigeons: Lea and Ryan, 1983). Another example of probable use of some absolute cues arises from an analysis of error trials of cebus monkeys required to categorise person and non-person slides. The authors of the study observed that misclassified non-person slides very often included a patch of reddish coloration, a feature occurring in about $1 / 3$ of person slides (D'Amato and van Sant, 1988). Therefore, the red hue appeared to be an absolute stimulus feature governing in part the monkey's classificatory behaviours.

\subsubsection{Template matching and feature analysis}

Rote learning, when it occurs, might be explained by the intervention of a template matching process, according to which subjects would have learned the correct response to each separate instance of the stimuli. In such a case, "identification of an object involves a matching process between a perceptual pattern and all the stored templates" (Roitblat and von Fersen, 1992, p. 701).

In fact, this hypothesis can be easily dismissed by introducing a transfer phase involving novel stimuli, i.e. stimuli for which no template is available. There is an abundant literature showing the abilities of animals from different species to successfully categorise to novel instances at their first presentations. On these experimental grounds as well as on other grounds (e.g. the enormous memory load involved in template matching), this process is very unlikely to account for categorical representations.

An alternative to template matching is feature analysis. According to this theory (Lea and Ryan, 1983), objects are characterised by a set of perceptual features. It is hypothesised that a subset of these features (differently combined) can be used by the animal as a basis for discrimination (Lea, 1984).

Several studies investigated feature learning in animals using artificial stimuli instead of natural scenes. For example, Morgan et al. (1976, with pigeons) and Schrier et al. (1984, with macaques) showed that their animals were using multiple features to categorise alphanumeric characters. In fact, feature theory permits to conclude that no single stimulus dimension is either necessary or sufficient to determine category membership (e.g. Dennis et al., 1973). Thus, researchers have as- 
sumed that natural as well as artificial categories (such as those just described) were based on the use of multiple or polymorphous features. For Lea and Harrison (1978, p. 535), a polymorphous concept formation implies an "ability to treat similarly a set of disparate stimuli not linked by any single, simple, feature, and to generalise from given instance, to new ones".

The use of polymorphous stimuli has been systematically studied in birds and mammals using artificial objects (e.g. compound stimuli made with a combination of shapes, colours and other dimensions of the stimuli). Typically, in this kind of experiments, subjects are first trained to classify two out of three feature stimuli. Then, transfer of performance is assessed with the prototypes of each category (for an example in pigeons, Lea and Harrison, 1978; for an example in monkeys, Jitsumori, 1994).

\subsubsection{Exemplar and prototype based theories}

Exemplar based models of categorisation have been popularised in the field of human cognition (e.g. Medin and Schaffer, 1978). Such theories hold that learned categories are stored in memory in a synthetical way (for example, under the form of prototypes; Rosch and Mervis, 1975).

Experimental evidence gathered so far with animals has not provided clear-cut demonstrations of the validity of prototype theories. Some experimental attempts to search for a prototype effect can, nevertheless, be reported. Such an effect is expressed by a better categorical performance with prototypical stimuli representing the central tendency of the categories than with other, less typical, exemplars. Thus, prototypical effects have been reported in studies with pigeons (von Fersen and Lea, 1990; Aydin and Pearce, 1994). However, in these studies the evidence of typicality effects is somewhat tempered by the previous associations between reinforcement and stimulus choice, given that the procedure involved the presentation of prototypical forms to subjects before testing. Moreover, several studies have reported no difference in the discrimination between prototype and non-prototype stimuli in birds (e.g. Watanabe, 1988; Huber and Lenz, 1993; Jitsumori, 1993; Lea et al., 1993).
Only two studies appear to have assessed prototype effects in nonhuman primates. Jitsumori (1994) trained rhesus monkeys with artificial stimuli defined by three two-valued positive or negative dimensions (colour, shape, and background colour). In transfer, both the prototypes and novel exemplars of the two categories were shown to the animals. Three from five monkeys demonstrated a high level of transfer with the prototypes. However, for those monkeys there was no statistical difference between the performance with prototypes and that obtained with the nonprototypical stimuli.

In another experiment, baboons were trained and tested with polymorphous artificial stimuli (Dépy et al., 1997). Results showed that the monkeys classified the prototypes more accurately than the other exemplars. However, the analysis of training performance indicated that their discriminations did not involve prototypical representations of the categories, but rather depended upon feature and exemplar-response associations. It was further hypothesised that the monkeys' better performance with the prototypes rested on peak shift and/or novelty effects (Hanson, 1959; see also, for similar findings and interpretations with human subjects, McLaren et al., 1995).

In brief, the state of knowledge in the animal (and human) literature calls for caution in concluding about the role of exemplar-based theories of categorisation. It is likely, as suggested by Roitblat and von Fersen (1992), that a better understanding of the complexity of categorisation behaviours calls for an alternative and more comprehensive theory that would incorporate feature analysis and prototype theories (e.g. relaxation theories).

\subsubsection{Procedural requirements and evidence of categorisation}

A final question must be asked when assessing categorical judgements and the use of a generalised concept matching in animal experiments. It concerns some procedural requirements that must be respected in order to ensure that subjects truly categorise and do not just respond on the basis of stimulus generalisation. In this respect, two procedural precautions must be singled out. 
Firstly, during transfer, test stimuli (usually novel ones) must be presented under extinction conditions in order to avoid associations between these stimuli and reinforcement. Alternatively, only the first trial of transfer must be retained to establish generalisation abilities. Secondly, a proper assessment of categorisation requires not only that subjects conceive that different objects have common class attributes, but also that the subjects can discriminate between individual members within a category (see also Wasserman et al., 1988; Thompson, 1995). Thus, in order to dismiss simple stimulus generalisation it must be demonstrated, in a first step, that the subject can discriminate between objects belonging to the different categories. The second step implies a demonstration that stimuli to be classified in the same category are discriminably different from one another. Very few studies have followed this two-step procedure, but when this issue has been addressed experimentally the results are informative. For example, although pigeons proved capable of performing between-class discriminations (i.e. of categorising oak leaves versus non-oak leaves), they appeared to be unable to perform within-class discriminations (Cerella, 1979). In the same vein, baboons were able to show high transfer in a matching-to-sample task implying categorisation of artificial stimuli. However, not all subjects exhibited accurate discrimination between the exemplars belonging to the same category (Vauclair and Fagot, 1996). Such a failure suggests some limited categorical abilities in the animals tested.

\section{Category formation of conspecifics and of social relationships}

So far category formation and some of its underlying cognitive processes have been discussed mainly with respect to physical objects or features. There is, however, another context in which animals elaborate natural categories: that of their relations with conspecifics. Given that comparative social cognition is admittedly a fascinating but immature field of research (Vauclair, 1996), the study of social categories should deci- sively contribute to it. Animals may represent the classes of their conspecifics, but also the classes of relationships established between conspecifics or between the latter and themselves. Accordingly, two kinds of social categories will be distinguished and discussed now.

\subsection{Social categories: recognition of conspecifics and identification of conspecific individuals}

Categories may be formed about entities and their indissociable properties, particularly their distinctive features accessible to perception. Social recognition bears relevance to category formation in animals, since it makes them distinguish between the natural classes of conspecifics and nonconspecifics, between members of various species, or between various classes of members of their own species (e.g. dominants and subordinates). Thus, pigeons trained to discriminate between pictures of a variety of animals detect pigeon-like stimuli and seem to form an abstract representation, or concept, of several animal species including their own (Poole and Lander, 1971; Herrnstein et al., 1976; Watanabe, 1992). In these tests as in others, carried out also with domestic chickens, the images of conspecifics and of nonconspecifics were those of socially unfamiliar individuals (Ryan and Lea, 1994). It is furthermore obvious, from naturalistic observations, that live birds readily distinguish between unfamiliar conspecifics and strangers of another species (Colgan, 1983), the same as they clearly recognise familiar conspecifics from acquainted individuals of other species. It is therefore reasonable to assert that in their real social life, animals use a general representation of conspecific-like objects whereby they reliably recognise individuals falling under such category. However, this may not be true in the context of concept discrimination experiments presenting photographs of unfamiliar individuals only, taking for granted that subjects should recognise there pictures of conspecifics. But, after all, these may be viewed just as complex artificial configurations devoid of any social significance. In order to rule out this possibility, visual discrimination experiments should compare the training and/or transfer performances in the following categories of pictures: 
Socially familiar conspecifics versus familiar non-conspecifics, expecting discriminations to be better for conspecifics (and similarly for the comparison between conspecific and non-conspecific strangers);

Socially familiar conspecifics versus conspecific strangers, expecting discriminations to be better in the case of familiarity (and similarly for the comparison between familiar and unfamiliar non-conspecifics).

The subjects might well make correct discriminations with pictures of unfamiliar individuals (Ryan, 1982), even of non-conspecifics (Ryan and Lea, 1994). However, the most accurate performances should be obtained with pictures of familiar conspecifics, to ascertain that animals recognise really social objects and that they form the category of all objects seen to possess the features typical of their own species. Presenting the pictures of several individuals in each set of photographs would enhance the prototypical character of the categories of conspecific and nonconspecific objects.

Individual recognition (of particular familiar conspecifics) is a specialised case of social category formation. In addition to several live observations, visual discrimination experiments have demonstrated that birds can learn to identify pictures of conspecifics of very similar appearance (Watanabe and Ito, 1991; Ryan and Lea, 1994). Subjects were first trained to discriminate the slides of two individuals and were subsequently presented with novel slides of the same conspecifics for the transfer tests. The results indicate that the polymorphous set of each individual's features becomes mentally represented as a single natural category, comprising the morphological properties unique to each bird. It is assumed that the subject constructs such an integrated representation, this unitary category being said to correspond to a concept. To speak of an individuality prototype, abstracted from visual information, would be preferable in the case of strictly individual attributes. Although empirical concepts originate in percepts, concepts are abstractions from particular entities or properties to their general counterparts, i.e. classes or attributes (Bunge, 1983). In the light of traditional philosophy, to speak of 'self-concept' would appear even more questionable (unless, of course, the subject suffers from severe dissociation of self-identity). Be that as it may, individual recognition is a complex operation involving perceptive classification of particular conspecifics, identified from degrees of (dis)similarity between their overlapping sets of properties (Zayan, 1994, for a set-theoretical model). Besides, the properties of conspecific individuals may be correctly identified despite great changes in their morphology, either in the course of ontogeny or following experimental procedures, notably when the novel transfer slides strongly differ from the training slides. But a more critical transformation is involved in these experiments (as well as in the above-mentioned species discrimination tests). It concerns the drastic alteration from real views of familiar conspecifics to photographic images of them and even more so when slides of strangers are shown, as was made in the most impressive experiments on individual discrimination tests. Thus, domestic cocks (Ryan, 1982) and hens (Ryan and Lea, 1994) correctly generalised visual information, acquired about socially unfamiliar conspecifics in training slides, to novel views of the same individuals in transfer slides. The same was found in pigeons, even though the novel pictures consisted of incomplete, or rotated, or scrambled, or even silhouettes of the training stimuli (Watanabe and Ito, 1991; Watanabe, 1992). Laying hens could be trained to discriminate between the pictures of two unknown chicks shown at the ages of 2 days, or 33 days, or 65 days. Some of the subjects were able to recognise novel slides of the same chicks pictured at ages different from those of the training views (intermediate but also extreme training ages; Ryan and Lea, 1990). In all these experiments, subjects certainly recognised the two target individuals as distinct perceptive categories, but not necessarily as individual conspecifics. The subjects might have perceived the slides as representing trifling chimeras, platonic dreams of a strange creature, at best as fictious conspecifics. To be really conclusive, such spectacular ability of birds to recognise images of strange conspecifics needs to be validated using views of individuals living in the group of the subject animals. Regrettably, 
control tests conducted so far with hens did not confirm recognition of socially familiar individuals when presented as slides. Thus, subjects trained to discriminate between slides of the heads of familiar and of unfamiliar conspecifics did not show significantly better discriminations when presented with novel slides of the familiar birds than with novel corresponding slides of the unfamiliar birds. As a general rule, the performances during this transfer phase were rather poor, perhaps because the novel slides presented strongly different views of the training birds. But there was also no evidence that subjects learned to discriminate the training slides of the familiar individuals more quickly than those of the unfamiliar ones (Bradshaw and Dawkins, 1993). Also disappointing are the results of a recent experiment (Dawkins, 1996); whereas hens recognise a flockmate from an unfamiliar conspecific even when just the head and neck are visible, such discrimination fails to be confirmed with photographs of the same target birds. Because transfer from live birds to pictures does not seem to occur (see also Trillmich, 1976), there is no evidence that hens see photographs or slides as representing real birds even if conspecifics were socially familiar. So, a picture of an individual hen may be perceived simply as an arbitrary pattern or as an array of salient features of a complex object, not necessarily social. It would, then, be pointless to show images only of arbitrary (unfamiliar) conspecifics in order to check that the head only or another part of the body permit social or individual recognition. Control tests should also be conducted with pictures of familiar conspecifics, unless an independent experiment has already shown that subjects do treat the slides as representatives of real birds.

Further experiments are required to examine whether there exists a correspondence between visual images of live animals and photographic views of them. One experiment appears to be precious in this respect and was carried out with longtailed macaques. Using a simultaneous two choice discrimination test, Dasser (1987) presented colour slides of the faces of two conspecifics which were living either in the subjects' group or as members of another colony. The subjects learned to discriminate the positive individuals of three pairs of familiar and of three pairs of unfamiliar stimuli. In subsequent transfer tests, they correctly matched novel slides (showing other views of the face but also views of other parts of the body) only if these represented the three familiar group members. Despite that only two subjects were tested, this finding gives a hint that animals can form consistent categories about particular individuals seen on slides as conspecifics. Here, visual recognition was surely facilitated by some stable relationships which existed between the subjects and the target individuals within the same colony. Thus, if a dominance hierarchy existed in the group, the subjects could also recognise the status (dominant or subordinate) of the familiar individuals towards other group members seen on the slides, and possibly also towards the subject animals themselves. In principle, animals should be able to represent and recognise social relations the same as they represent and recognise categories of conspecifics (e.g. dominants and subordinates) related by these bonds. Dasser (1988) demonstrated that macaque females correctly matched slides of infants to the slides of their mothers in numerous parent-infant pairs representing real bonds formed in the same colony. They certainly have recognised the particular mother-infant bonds, as well as the identity of the mothers and of the infants. From such recognition, it can be admitted that the subjects have abstracted to the general classes of mothers, of infants, and of mother-infant relations existing in the group. But nothing allows to conclude that the subjects formed the social concept of parentoffspring bond in general (as the author believes); i.e. nothing proves that they represented the category of a social bond beyond the realm of their particular group. What is doubtful is whether animals can form not only perceptive categories or concepts, but also conceptual categories of their conspecifics and of their social bonds. For example, from the categories of 'ideal' dominant and subordinate individuals not seen in any group, an animal would attain the concepts of dominance, of subordination, and of dominance relations in general. On the basis of such abstract (proto)types, the animal could, on the other hand, 
later 'recognise' real unfamiliar individuals apparently corresponding to these general attributes. In the case e.g. of a dominant conspecific, domestic hens or cocks, for example would distinguish individuals having large and colourful combs, having prior access to food, threatening all others, etc. These social concepts would correspond to unobservable properties the animal attributes to categories of virtual conspecifics (or animals in general) and to the categories of their possible social orderings. In the case of a linear dominance hierarchy, the properties would be asymmetry and transitivity of the order relations. To construct the concept of a linear dominance order, an animal would have to represent the structure of a hierarchy independently of the dominance relations it perceives in its stable group. The animal should also be able to infer transitivity by means of an operation analogous to logical deduction. These two cognitive abilities will be examined in the following section.

\subsection{Social categories: representation of transitivity in dominance hierarchies}

Transitivity may be viewed as a general or as a specific property of order relations. In the former case, it is a concept abstracted from various kinds of order relations perceived among objects and properties of various natural classes (or even among concepts and propositions, in which case it is a formal construct rather than an empirical concept; Bunge, 1983). In the latter case, transitivity is confined to single classes of objects and properties. Here we shall restrict it to a certain kind of dominance-subordination relation, namely one resulting from agonistic encounters among the members of a stable group, because it was investigated as such in the only available experiment touching upon the problem. It was conducted by Dasser (1987; unpublished; summarised by Vauclair, 1996, pp. 90-91 and by Zayan, 1994, pp. 241-242), with one female longtailed macaque to which were presented slides of familiar group members.

For the training trials of a simultaneous two choice discrimination test, the subject saw successive pairs of slides of the same three females holding in their group a transitive dominance order $\mathrm{A}>\mathrm{B}>\mathrm{C}$. On each trial, $\mathrm{B}$ was matched either to $\mathrm{A}$ or to $\mathrm{C}$, the choice for the dominant member of the pair being reinforced. For the transfer test the subject was presented with pairs of novel slides of 16 other group members (pairs of females but also of males). A different pair was always presented on each of 28 trials, and the social rank of 10 individuals was reversed at least once before the test was completed, thus precluding the possibility that the subject would learn always to choose a particular individual no matter which other it was paired with. The results reveal that the subject made a significant majority of correct choices for the dominant member of a pair.

The results are difficult to interpret. They surely demonstrate that the subject correctly recognised the dominance-subordination relationships existing in the group and by the same token, the identity of the various group members selected to be presented. They also indicate that the subject has formed and preserved a consistent representation of a property of the dominance order, namely asymmetry. The subject presumably has formed a perceptive or empirical concept of social asymmetry. This surely goes beyond the mere recognition of the relata, for the asymmetry had to be attributed to pairs of individuals which appeared more than once with a different status in the novel slides. However, nothing guarantees that the subject had not already seen the changes in dominance relations as these individuals alternately encountered superior and subordinate group members. Even if the subject had not seen the direction of all the dominance relations later presented during the experiment, nothing proves that it formed an abstract concept of asymmetric dominance, liable to be generalised to unfamiliar conspecifics. As to the transitive property of these dominance relations, this experiment cannot tell if the subject has deduced it from the set of pairs of individuals presented in succession. The animal would have to infer it for pairs of stimuli never presented, but mentally represented, as possible components of triads $(\mathrm{A}>\mathrm{C}$ being imagined after $\mathrm{B}<\mathrm{A}$ and $\mathrm{B}>\mathrm{C}$ ). Again, nothing proves that the subject had not seen in the group the third dyadic 
relation confirming the 'logical' connection with the dominance relations observed in the two antecedent dyads serving as premises. For example, the subject could have seen:

(1) $\mathrm{B}>\mathrm{C}$ or $\mathrm{C}>\mathrm{B}$, after $\mathrm{A}>\mathrm{B}$ and $\mathrm{A}>\mathrm{C}$;

(2) $\mathrm{A}>\mathrm{C}$ or $\mathrm{C}>\mathrm{A}$, after $\mathrm{A}>\mathrm{B}$ and $\mathrm{B}<\mathrm{C}$;

(3) $\mathrm{A}>\mathrm{C}$, after $\mathrm{A}>\mathrm{B}$ and $\mathrm{B}>\mathrm{C}$;

(4) $\mathrm{C}>\mathrm{B}$, after $\mathrm{A}>\mathrm{B}$ and $\mathrm{A}<\mathrm{C}$.

Here the animal would simply recognise, i.e. mentally reconstruct, a transitive dominance order among highly familiar conspecifics. To conclude, this experiment cannot demonstrate that animals generate a transitive order, inferring e.g. that $\mathrm{A}>\mathrm{C}$ from knowledge that $\mathrm{A}>\mathrm{B}$ and $\mathrm{B}>\mathrm{C}$ in the group. A fortiori, no experiment has yet indicated that animals represent social transitivity as a conceptual category, where $\mathrm{A}>\mathrm{C}$ would be inferred or predicted on a purely abstract basis and where the transitive link would hold true for dominance relations in general, not only for an agonistic or other kind of dominance settled among conspecifics.

Experiments could be designed to indicate whether an animal really constructs a representation of virtually transitive dominance relations, either between group members which have not interacted yet, or between itself and group members which it has not yet encountered. There is already good evidence that animals observe agonistic interactions between group members and attend the dominance outcomes of paired contests in a way that incites or inhibits their aggression towards the subordinate and the dominant member of a pair, respectively (Hogue et al., 1996). Visual information gained about the outcomes of initial contests may explain the fact that in small groups of various animal species transitive hierarchies occur as a typical pattern, often much more frequently than would be expected merely by chance. In triads of domestic chickens, dominance hierarchies of a linear structure (i.e. both complete and transitive) nearly always occur, whereas their theoretical probability is only of $P=0.75$ in animals meeting for the first time and having equal chances to win or to lose each agonistic encounter (Chase, 1982). A kind of social inference may account for linearity being so systematically recorded in triads of unfamiliar opponents. Admitting that in all cases the initial contest results in the dominance of A towards B, transitivity is guaranteed if $\mathrm{A}$ is dominant again after its second encounter, with $\mathrm{C}$ (double dominance of $\mathrm{A}$, see case 1 above) and also if $\mathrm{B}$ is dominated again after its encounter with $\mathrm{C}$ (double subordinance of $\mathrm{B}$, see case 2 above). In contrast, transitivity can occur as frequently as intransitivity, or circularity, either when B is first dominated by A but afterwards dominates $\mathrm{C}$ (case 3 above, $\mathrm{A}>\mathrm{C}$ being transitive but $\mathrm{C}>\mathrm{A}$ circular), or when A first dominates $\mathrm{B}$ but is afterwards defeated by $\mathrm{C}$ (case 4 above, $\mathrm{C}>\mathrm{B}$ being transitive but $\mathrm{B}>\mathrm{C}$ circular). Transitivity will be strongly compelled if after their initial encounter, $\mathrm{A}$ and $\mathrm{B}$ can observe the outcome of their respective contest with $\mathrm{C}$. Thus, in the first case A would see its subordinate $\mathrm{B}$ dominate $\mathrm{C}$ and would conclude that it would itself a fortiori dominate $\mathrm{C}$ (inciting $\mathrm{A}$ to quickly attack $\mathrm{C}$ ). Supposing that $\mathrm{C}$ also could have observed the initial dominance of $\mathrm{A}$ over $\mathrm{B}$, then $\mathrm{C}$ would conclude to its very probable subordinance towards $\mathrm{A}$ and would at once tend to initiate retreat instead of challenging A. In the second case, B would see its dominant A being defeated by $\mathrm{C}$ and would conclude that it would a fortiori itself be defeated by C (hence B's likely decision to withdraw). Meanwhile, having observed the initial subordinance of B towards A, C would conclude to its very probable own dominance over $\mathrm{B}$ and would be incited to attack B at once. In both cases, the frequency of a transitive order would be greatly increased, the same as would be the frequency of complete dominance orders. Hence the high frequency of linear hierarchies among triads of chickens and among component triads of larger groups (Chase, 1982).

Such cognitive process may be termed perceptive inference of social transitivity. The transitive inference which anticipates the most probable outcomes at the third contest operates upon a combination of visual information, gained about the status of two conspecifics, and of the observer's own dominance or subordination experience with one of the conspecifics. Short-term recognition of the visually and/or socially familiar individuals must be involved too in the prediction of 
the transitive outcome, whereas long-term individual recognition should strengthen the stability of the linear dominance in small groups.

Very little is known about social transitivity in animals, as is made obvious in the review by Delius and Siemann (this issue). Their paper also emphasises that while ordering physical objects or configurations, pigeons or primates might not necessarily operate by transitive inference even when showing correct order preferences. These may result not from logical deduction but from a simpler discrimination process, where suitable training ensures generalisation of the order direction during the transfer phase testing for transitivity. Concerning social transitivity, Cheney and Seyfarth (1990) very honestly concede that the data from natural groups of primates are coarse and that field observations suggest, but never have demonstrated, that primates infer the structure of their dominance hierarchy from observation of interactions among group members. The fact that agonistic encounters result in apparently transitive orders does not prove that animals have in mind a representation of transitivity. Thus, B may know and continuously recognise its dominant $\mathrm{A}$ and its subordinate $\mathrm{C}$, but without actually knowing that $\mathrm{A}$ dominates $\mathrm{C}$ unless and until $\mathrm{B}$ sees it. Similarly, B would need to see that $\mathrm{C}$ dominates $\mathrm{E}$, after having observed the successive dominances of $\mathrm{C}$ over $\mathrm{D}$ and of $\mathrm{D}$ over $\mathrm{E}$, to achieve a mental representation of transitivity. An inference proper requires the animal to construct putative transitive orders on the basis of incomplete social information, ignoring the dominance relations among some of the possible pairs of group members. In this case, the animal would anticipate dominance outcomes not yet seen. Here, social transitivity would be tacitly assumed and explicitly predicted. Inference of a group's linear structure would, in addition, require the animal to conceive transitivity for all dominance relations whereas only a subset of them have been seen to be transitive. An animal could, having seen and memorised the dominance outcomes of successive paired encounters, limit its representation of a transitive order to the subset of group members it had the opportunity to observe interacting. Then, the animal would proceed not by transitive inference but by associative transitivity, as Cheney and Seyfarth (1990) have specified, borrowing a distinction made by D'Amato and coworkers in some of their experiments on transitive ordering of physical objects in primates. Here, associative transitivity corresponds to an internal representation of the sequential order among a series of stimuli; the ordering requires simply some prior association between particular training stimuli, e.g. items $\mathrm{A}$ and $\mathrm{C}$, which become related but not according to a relational rule common to the antecedent pairs of stimuli ( $\mathrm{A}-\mathrm{B}$ and $\mathrm{B}-\mathrm{C})$. In a group, animals may consistently form representations about the relations only of the individuals they have seen to interact at high rates. Thus, A may be constantly seen to attack and dominate all group members $\mathrm{B}, \mathrm{C}, \mathrm{D}$ and $\mathrm{E}$; contrarily, $\mathbf{J}$ may be seen to be continuously attacked and defeated by F, G, H and I. An animal could, on the basis of such a clear asymmetry and not having seen any interaction between $\mathrm{A}$ and $\mathrm{J}$, spontaneously conclude tht A would most probably dominate J. No deductive inference proper would be involved to represent a transitive link.

Not a single experiment permits to assume that animals mentally construct the category of a linear dominance hierarchy on a perceptive basis, let alone on a purely conceptual one. The data gathered hitherto from field observations or from learning discrimination experiments are tiny and leave us with little more than conjectures. As to concept discrimination experiments conducted to test for category formation of conspecifics, their results are equivocal and need to be complemented by appropriate controls.

The study of social categories in animals has been considerably neglected by current research on comparative cognition. Accordingly, room was made for it in the present volume, where it appears implicitly or as a specific concern, including in the context of human social psychology where this topic has become of paramount importance. 


\section{Acknowledgements}

René Zayan is very much indebted to Dominique Domken for his fruitful discussions and collaboration, to Jacques Beaugrand for mutual interest in social cognition, and to Yves Lamberty and Xavier Seron for personal support.

\section{References}

Aydin, A., Pearce, J.M., 1994. Prototype effects in categorization by pigeons. J. Exp. Psychol.: Anim. Behav. Process. 20, 264-277.

Bradshaw, R.H., Dawkins, M.S., 1993. Slides of conspecifics as representatives of real animals in laying hens (Gallus domesticus). Behav. Proc. 28, 165-172.

Bunge, M. (Ed.), 1983. Treatise on Basic Philosophy, vol. 5. Epistemology and Methodology I.D., Reidel, Dordrecht.

Cerella, J., 1979. Visual classes and natural categories in the pigeon. J. Exp. Psychol.: Hum. Perc. Perf. 5, 68-77.

Chase, I.D., 1982. Dynamics of hierarchy formation: the sequential development of dominance relationships. Behaviour 80 , 218-240.

Cheney, D.L. Seyfarth, R.M., 1990. How Monkeys see the World. University of Chicago Press, Chicago.

Colgan, P.W., 1983. Comparative Social Recognition. Wiley, New York.

Cook, R., Cavoto, K.K., Cavoto, B.R., 1995. Same-different texture discrimination and concept learning by pigeons.. J. Exp. Psychol.: Anim. Behav. Process. 21, 253-260.

Crystal, J.D., Shettleworth, S.J., 1994. Spatial list learning in black-capped chickadees. Anim. Learn. Behav. 22, 77-83.

D'Amato, M.R., van Sant, P., 1988. The person concept in monkeys (Cebus apella). J. Exp. Psychol.: Anim. Behav. Process. 14, 43-55.

D’Amato, M.R., Salmon, D.P., Colombo, M., 1985. Extent and limits of the matching concepts in monkeys (Cebus apella). J. Exp. Psychol.: Anim. Behav. Process. 11, 35-51.

Dasser, V., 1987. Slides of group members as representations of the real animals (Macaca fascicularis). Ethology 76, 65-73.

Dasser, V., 1988. A social concept in Java monkeys. Anim. Behav. 36, 225-230.

Dawkins, M.S., 1996. Distance and social recognition in hens: implications for the use of photographs as social stimuli. Behavior 33, 663-680.

Delius, J.D., 1992. Categorical discrimination of objects and pictures by pigeons. Anim Learn. Behav. 20, 301-311.

Delius, J.D., Siemann, 1998. Transitive responding in animals and humans: Exaptation rather than adaptation? Behav. Proc. 42, 107-137.

Dennis, I., Hampton, J.A., Lea, S.E.G., 1973. New problem in concept formation. Nature 243, 101-102.

Dépy, D., Fagot, J., Vauclair, J., 1997. Categorization of three-dimensional stimuli by humans and baboons: search for prototype effects. Behav. Process. 39, 299-306. von Fersen, L., Lea, S.E.G., 1990. Category discrimination by pigeons using five polymorphous features. J. Exp. Anim. Behav. 54, 69-89.

Gardner, R.A., Gardner, B.T., 1984. A vocabulary test for chimpanzees (Pan troglodytes). J. Comp. Psychol. 98, 381404.

Hanson, H.M., 1959. Effects of discrimination training on stimulus generalization. J. Exp. Psychol. 58, 321-334.

Harnad, S. (Ed.), 1987. Perception: the groundwork of cognition. Cambridge University Press, New York.

Herman, L.M., Hovancik, J.R., Gory, J.D., Bradshaw, G.L., 1989. Generalization of matching by a bottlenosed dolphin (Tursiops truncatus): Evidence for invariance of cognitive performance with visual and auditory materials. J. Exp. Psychol.: Anim. Behav. Process. 15, 124-136.

Herrnstein, R. J., 1990. Levels of stimulus control: a functional approach. Cognition 37, 133-166.

Herrnstein, R.J., Loveland, D. H., 1964. Complex visual concept in the pigeon. Science 146, 149-151.

Herrnstein, R.J., Loveland, D.H., Cable, C., 1976. Natural concept in pigeons. J. Exp. Psychol.: Anim. Behav. Process. 2, 285-311.

Hogue, M.E., Beaugrand, J.P., Lagüe, P.C., 1996. Coherent use of information by hens observing their former dominant defeating or being defeated by a stranger. Behav. Process. 38, 241-252.

Huber, L., Lenz, R., 1993. A test of the linear feature model of polymorphous concept discrimination with pigeons. Q. J. Exp. Psychol. 46B, 1-18.

Jitsumori, M., 1993. Category discrimination of artificial polymorphous stimuli based on feature learning. J. Exp. Psycol. Process.: Anim. Behav. 19, 244-254.

Jitsumori, M., 1994. Discrimination of artificial polymorphous categories by rhesus monkeys (Macaca mulatta). Q. J. Exp. Psychol. 47B, 371-386.

Kamil, A.C., Balda, R.P., 1985. Cache recovery and spatial memory in Clark's nutcrackers (Nucifraga columbiana). J. Exp. Psychol. Process.: Anim. Behav. 11, 95-111.

Lea, S.E.G. 1984. In what sense do pigeons learn concepts? In: Roitblat, H.L., Bever, T.G., Terrace, H.S. (Eds.), Animal Cognition, Lawerence Erlbaum, Hillsdale, NJ, pp. 263276.

Lea, S.E.G., Harrison, S.N., 1978. Discrimination of polymorphous stimuli sets by pigeons. Q. J. Exp. Psychol. 30, 521-537.

Lea, S.E.G., Ryan, C.M.E., 1990. Featural analysis of pigeons' acquisition of discrimination between letters. In: Commons, M.L., Herrnstein, R.J., Wagner, A.R. (Eds.), Quantitative Analyses of Behaviour, vol. 4. Ballinger, Cambridge, MA, pp. 239-253.

Lea, S.E.G., Ryan, C.M.E., 1990. Unnatural concepts and the theory of concept discrimination in birds. In: Commons, M.L., Herrnstein, R.J., Kosslyn, S., Mumford, D. (Eds.), Quantitative Analyses of Behaviour, vol. 8. Lawrence Erlbaum, Hillsdale, NJ, pp. 165-185.

Lea, S.E.G., Lohmann, A., Ryan, C.M.E., 1993. Discrimination of five-dimensional stimuli by pigeons: limitations of feature analysis. Q. J. Exp. Psychol. 46, 19-42. 
McLaren, I.P.L., Bennett, C.H., Guttman-Nahir, T., Kim, K., Mackintosh, N.J., 1995. Prototype effects and peak shift in categorization. J. Exp. Psychol.: Learn. Mem. Cogn. 21, 662-673.

Medin, D.L., Schaffer, M.M., 1978. Context theory of classification learning. Psycol. Rev. 85, 207-238.

Morgan, M.J., Fitch, M.D., Holman, J.G., Lea, S.E., 1976. Pigeons learn the concept of an 'A'. Perception 5, 57-66.

Neiworth, J.J., Wright, A.A., 1994. Monkeys (Macaca mulatta) learn category matching in a nonidentical same-different task. J. Exp. Psychol.: Anim. Behav. Process. 20, 429-435.

Oden, D.L., Thompson, R.K.R., Premack, D., 1988. Spontaneous transfer of matching by infant chimpanzees (Pan troglodytes). J. Exp. Psychol. Process.: Anim. Behav. 14, $140-145$.

Oden, D.L., Thompson, R.K.R., Premack, D., 1990. Infant chimpanzees (Pan troglodytes) spontaneously perceive both concrete and abstract same/different relations. Child Dev. 61, 621-631.

Poole, J., Lander, D.G., 1971. The pigeons' concept of a pigeon. Psychon. Sci. 25, 157-158.

Premack, D., 1983. The codes of man and beast. Behav. Brain Sci. 6, 125-167.

Roberts, W.A., Mazmanian, D.S., 1988. Concept learning at different levels of abstraction by pigeons, monkeys and people. J. Exp. Psychol. Process.: Anim. Behav. 14, $247-$ 260.

Roitblat, H.L., von Fersen, L., 1992. Comparative cognition: Representations and processes in learning and memory. Annu. Rev. Psychol. 43, 671-710.

Rosch, E., Mervis, C.B., 1975. Family resemblances: studies in the internal structure of categories. Cogn. Psychol. 7, $573-605$

Ryan, C.M.E., 1982. Concept formation and individual recognition in the domestic chicken (Gallus gallus). Behav. Anal. Lett. 2, 213-220.

Ryan, C.M.E., Lea, S.E.G., 1990. Pattern recognition, "updating" and filial imprinting in the domestic chicken (Gallus gallus). In: Commons, M.L., Herrnstein, R.J., Kosslyn, S., Mumford, D. (Eds.), Quantitative Analysis of Behaviour, vol. 8, Lawrence Erlbaum, Hillsdale, NJ, pp. 89-110.

Ryan, C.M.E., Lea, S.E.G., 1994. Images of conspecifics as categories to be discriminated by pigeons and chickens: slides, video tapes, stuffed birds and live birds. Behav. Process. 33, 155-176.

Savage-Rumbaugh, E.S., Rumbaugh, D.M., Smith, S.T., Lawson, J., 1980. Reference: the linguistic essential. Science 210, $921-925$.

Schrier, A.M., Angarella, R., Povar, M., 1984. Studies of concept formation by stumptail monkeys: concepts monkeys, humans and letter A. J. Exp. Psychol. Process.:
Anim. Behav. 10, 564-584.

Thompson, R.K.R., Oden, D.L., 1996. A profound disparity revisited. Perception and judgement of abstract identity relations by chimpanzees, human infants and monkeys. Behav. Process. 35, 149-161.

Thompson, R.K.R., 1995. Natural and relational concepts in animals. In: Roitblat, H., Meyer, J.A. (Eds.), Comparative Approaches to Cognitive Science. MIT Press, Cambridge, pp. $175-224$.

Thompson, R.K.R., Oden, D.L., Boysen, S.T., 1997. Language-naive chimpanzees (Pan troglodytes) judge relations between relations in a conceptual matching-to-sample task. J. Exp. Psychol. Process.: Anim. Behav. 23, 31-43.

Trillmich, F., 1976. Learning experiments on individual recognition in Budgerigars (Melopsittacus undulatus). Z. Tierpsychol. 41, 372-395.

Tyrrell, D.J., Stauffer, L.B., Snowman, L.G., 1991. Perception of abstract identity/difference relationships by infants. Inf. Behav. Dev. 14, 125-129.

Vauclair, J., 1996. Animal Cognition. An Introduction to Modern Comparative Psychology. Harvard University Press, Cambridge-London.

Vauclair, J., Fagot, J., 1996. Categorization of alphanumeric characters by baboons (Papio papio): within and between class stimulus discrimination. Curr. Psychol. Cogn. 15, 449-462.

Vaughan, W. Jr., Greene, S.L., 1984. Pigeon visual memory capacity. J. Exp. Psychol. Process.: Anim. Behav. 10, 256271.

Wasserman, E.A., Kiedinger, R.E., Bhatt, R.S., 1988. Conceptual behavior in pigeons: Categories, subcategories and pseudocategories. J. Exp. Psychol. Process.: Anim. Behav. 14, 235-246.

Watanabe, S., 1988. Failure of visual prototype learning in the pigeon. Anim. Learn. Behav. 16, 147-152.

Watanabe, S., 1992. Effects of lesions in the ectostriatum and Wulst on species and individual discrimination in pigeons. Behav. Brain Res. 49, 197-203.

Watanabe, S., Lea, S.E.G., Dittrich, W.H., 1993. What can we learn from experiments on pigeon concept discrimination? In: Bischof, H.J., Zeigler H.P. (Eds.), Avian Vision and Cognition. MIT Press, Cambridge, pp. 352-376.

Watanabe, S., Ito, Y., 1991. Discrimination of individuals in pigeons. Bird Behav. 9, 20-29.

Yoshikubo, S., 1985. Species discrimination and concept formation by rhesus monkeys (Macaca mulatta). Primates 26, 285-299.

Zayan, R., 1994. Mental representation in the recognition of conspecific individuals. Behav. Process. 33, 233-246.

Zentall, T.R., Hogan, D.E., 1976. Pigeons can learn identity or difference, or both. Science 191, 408-409. 